\title{
High-quality thin-film passivation by catalyzer-enhanced chemical vapor deposition for organic light-emitting diodes
}

\author{
Han-Ki Kim ${ }^{\text {a) }}$ \\ Department of Information and Nano Materials Engineering, Kumoh National Institute of Technology \\ (KIT), 1 Yangho-dong, Gumi, Gyeongbuk 730-701, Korea \\ Myung Soo Kim \\ Core Technology Laboratory, Samsung SDI, Co., Ltd., 575 Shin-dong, Youngtong-gu, Suwon, Gyeonggi-do \\ 442-391, Korea \\ Jae-Wook Kang and Jang-Joo Kim \\ School of Materials Science and Engineering, Seoul National University, Silim-dong, Seoul 151-741, Korea \\ and Organic Light Emitting Diodes Center, Seoul National University, Silim-dong, Seoul 151-741, \\ Korea \\ Min-Su Yi \\ Department of Materials Science and Engineering, Sangju National University, Sangju, Gyeongbuk \\ 742-711, Korea
}

(Received 19 September 2006; accepted 29 November 2006; published online 2 January 2007)

The thin-film passivation of organic light-emitting diodes (OLEDs) by a $\mathrm{SiN}_{x}$ film grown by catalyzer-enhanced chemical vapor deposition was investigated. Using a tungsten catalyzer connected in series, a high-density $\operatorname{SiN}_{x}$ passivation layer was deposited on OLEDs and bare polycarbonate (PC) substrates at a substrate temperature of $50{ }^{\circ} \mathrm{C}$. Despite the low substrate temperature, the single $\mathrm{SiN}_{x}$ passivation layer, grown on the PC substrate, exhibited a low water vapor transmission rate of $(2-6) \times 10^{-2} \mathrm{~g} / \mathrm{m}^{2} /$ day and a high transmittance of $87 \%$. In addition, current-voltage-luminescence results of an OLED passivated with a 150-nm-thick $\mathrm{SiN}_{x}$ film compared to nonpassivated sample were identical indicating that the performance of an OLED is not critically affected by radiation from tungsten catalyzer during the $\mathrm{SiN}_{x}$ deposition. Moreover, the lifetime to half initial luminance of an OLED passivated with the single 150-nm-thick $\mathrm{SiN}_{x}$ layer was 2.5 times longer than that of a nonpassivated sample. (C) 2007 American Institute of Physics. [DOI: $10.1063 / 1.2425021]$

Organic light-emitting diodes (OLEDs) have recently entered the display market as sub- and main window displays for cellular phones due to their extremely thin thickness, high peak brightness, high dark room contrast, low power consumption, low cost, superviewing ability, and fast response time. ${ }^{1}$ However, the long-term stability of OLEDs is still limited due to the instability of the luminescent organic materials, interfacial reactions, and chemical reactions of the organic layers with oxygen and moisture in the air. ${ }^{2,3}$ In particular, organic materials are very sensitive to moisture and oxygen when exposed to ambient air. Although lid type encapsulation is typically used in OLEDs, it comes with its own set of problems such as the penetration of oxygen and moisture through epoxy resin, an increase in thickness, a complicated process, and difficulty of application to flexible OLEDs. To achieve further advances in the production of OLEDs and flexible displays, it will be necessary to develop high-quality thin-film passivation with a low water vapor transmission rate (WVTR), excellent reliability, long-term stability and lifetime, and a high degree of transparency. Transparent $\mathrm{SiN}_{x}, \mathrm{SiO}_{x}, \mathrm{SiO}_{x} \mathrm{~N}_{y}, \mathrm{AlO}_{\mathrm{x}}$, and $\mathrm{Al}_{2} \mathrm{O}_{3}: \mathrm{N}$ films are currently employed as inorganic passivation layers for OLEDs. ${ }^{4-8}$ However, a single inorganic thin film is not sufficiently dense to protect the OLEDs from permeation by moisture and oxygen. Therefore, multilayered passivation

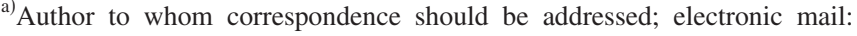
hkikim@kumoh.ac.kr composed of inorganic/inorganic or polymer/inorganic (Barix coating) layers has been proposed. ${ }^{5,9}$ However, most reported deposition techniques for inorganic films are based on a plasma process which is harmful to organic materials. While the effect of the exposure OLEDs to plasma is well recognized, the development of a plasma-free thin-film passivation process is still lacking. ${ }^{10}$

In this work, we report on a plasma-free thin-film passivation technique for OLEDs and flexible OLEDs using a catalyzer-enhanced chemical vapor deposition (CECVD) system. Despite the low substrate temperature used, a single 150-nm-thick $\mathrm{SiN}_{x}$ layer grown on a Polycarbonate (PC) substrate showed a low WVTR of $(2-6) \times 10^{-2} \mathrm{~g} / \mathrm{m}^{2} /$ day and a high transmittance of $87 \%$. The current-voltageluminescence $(J-V-L)$ characteristics of the OLED passivated with the $\operatorname{SiN}_{x}$ layer were identical to a nonpassivated sample, indicating no thermal damage by tungsten catalyzer. In addition, the increased lifetime of the OLED passivated by the $\mathrm{SiN}_{x}$ layer indicates that CECVD is a promising plasma-free passivation technique that can be used in place of the conventional plasma-enhanced CVD (PECVD) and inductively coupled plasma CVD (ICP-CVD) processes.

To investigate the effect of thin-film passivation on OLEDs, we prepared phosphorescent OLEDs. All organic layers were grown on an indium tin oxide (ITO) anode by thermal evaporation at $1 \times 10^{-7}$ Torr in the following order: hole transporting layer (40 $\mathrm{nm} \alpha$-napthylphenlylbiphenyl (NPB)/emission layer [30 nm 4,4'-bis(9-carbazolyl)biphenyl $\left.(\mathrm{CBP})+6 \% \operatorname{Ir}(\mathrm{ppy})_{3}\right] /$ hole blocking layer $[10 \mathrm{~nm}$ 


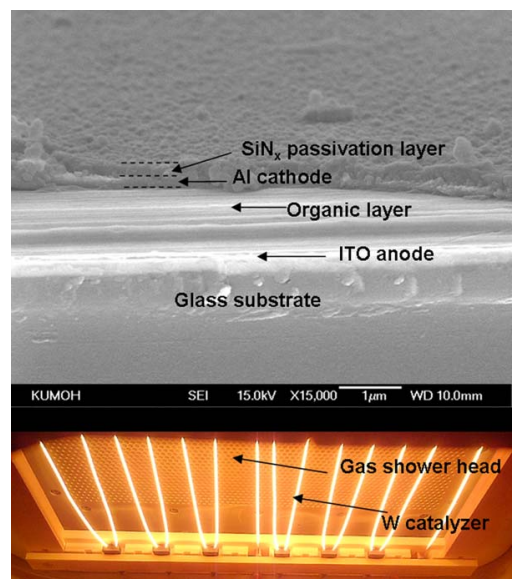

FIG. 1. (Color online) (a) Cross sectional SEM image of an OLED passivated with a $\mathrm{SiN}_{x}$ layer grown by CECVD and (b) the tungsten catalyzer in CECVD system.

2,9-dimethyl-4,7-diphenyl-1,10-phenanthroline (BCP)] electron transporting layer [40 $\mathrm{nm}$ tris-(8-hydroxyquinoline) aluminum $\left(\mathrm{Alq}_{3}\right)$ ]. Finally, a 1-nm-/100-nm-thick LiF/Al cathode layer was patterned using a shadow mask for an active device area of $3 \mathrm{~mm}^{2}$. After preparation of the OLED samples, they were passivated with a $\mathrm{SiN}_{x}$ layer grown using a CECVD system. Figure 1(a) shows a cross sectional scanning electron microscopy (SEM) image of an OLED passivated with a $150-\mathrm{nm}$-thick $\mathrm{SiN}_{x}$ layer. For the deposition of a high-density $\operatorname{SiN}_{x}$ passivation layer at a low substrate temperature, a specially designed CECVD system with a tungsten catalyzer and a bipolar electrostatic chucking (BEC) system were employed. The ladder-shaped tungsten catalyzer with a diameter of $0.7 \mathrm{~mm}$ and size of $45 \times 50 \mathrm{~cm}^{2}$ was located below the showerhead, as shown in Fig. 1(b). This tungsten catalyzer plays an important role in catalytically decomposing $\mathrm{SiH}_{4}, \mathrm{NH}_{3}$, and $\mathrm{H}_{2}{ }^{11}$ A mixture of $\mathrm{SiH}_{4}, \mathrm{NH}_{3}$, and $\mathrm{H}_{2}$ was used for the deposition of the $\mathrm{SiN}_{x}$ passivation layer. Optical transmittance through the $\operatorname{SiN}_{x}$ film grown by CECVD was measured in the wavelength range from 220 to $800 \mathrm{~nm}$. To investigate structural properties of the $\mathrm{SiN}_{x}$ film, synchrotron x-ray scattering examination was carried out. The WVTR value for $\mathrm{SiN}_{x}$ films grown on PC substrates $\left(10 \times 10 \mathrm{~cm}^{2}\right)$ was determined at $38 \pm 2{ }^{\circ} \mathrm{C}, 100 \% \mathrm{RH}$ by MOCON for $72 \mathrm{~h}$. After deposition of the $\mathrm{SiN}_{x}$ passivation layer on the OLED, the $J-V-L$ characteristics of OLEDs were measured using a photo research PR-650 spectrophotometer driven by a programmable dc source. Driven at $2 \mathrm{~mA} / \mathrm{cm}^{2}$, the device lifetime of OLED passivated with the $\mathrm{SiN}_{x}$ film at an initial luminance of $500 \mathrm{~cd} / \mathrm{m}^{2}$ was measured.

Figure 2 shows typical $\theta-2 \theta$ scan result along the surface normal direction in reciprocal space $[Q$ $=4 \pi \sin (2 \theta / 2) / \lambda]$ and transmittance spectra of the 150-nm-thick $\mathrm{SiN}_{x}$ film grown in a hydrogen ambient $\left[\mathrm{SiH}_{4} / \mathrm{NH}_{3} / \mathrm{H}_{2}=32 / 4800 / 1500 \mathrm{SCCM}\right.$ (sccm denotes cubic centimeter per minute at STP)]. The diffraction profiles of the $\mathrm{SiN}_{x}$ film exhibit one broad peak, characteristic of an amorphous structure. Regardless of the working pressure and ratio of reactive gas flow, all of the $\mathrm{SiN}_{x}$ samples grown on glass substrates show a similar amorphous structure due to the low substrate temperature maintained by the BEC system. The amorphous structure of the $\mathrm{SiN}_{x}$ film indicates that the effect of the tungsten catalyzer $\left(\sim 1800^{\circ} \mathrm{C}\right)$ on the sub-

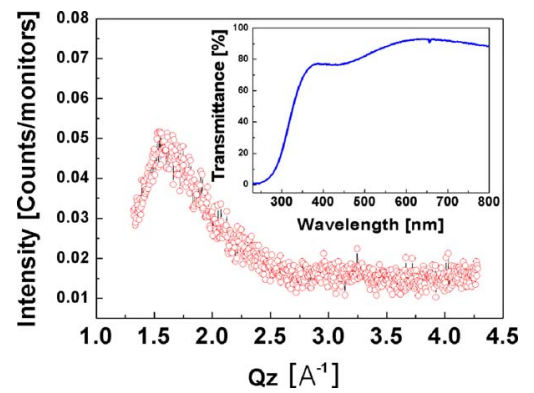

FIG. 2. (Color online) Synchrotron x-ray scattering result for $\operatorname{SiN}_{x}$ film deposited by CECVD on a glass substrate at optimized condition with the transmittance of the $\mathrm{SiN}_{x}$ in the inset.

strate during $\mathrm{SiN}_{x}$ growth is completely eliminated by the BEC. It has been reported that a high hydrogen dilution ratio in a catalytic CVD resulted in the transition of the microstructure of a Si film from amorphous to polycrystalline. ${ }^{11,12}$ However, unlike Si film, even though high hydrogen flow ratio was used, the $\mathrm{SiN}_{x}$ film grown by CECVD had a completely amorphous structure due to the effect of the BEC system. It is noteworthy that the transmittance of the $\mathrm{SiN}_{x}$ film in the green region is $87 \%$ which is similar to that of glass substrate, as shown in the inset of Fig. 2.

To measure the WVTR of the $\mathrm{SiN}_{x}$ film grown by CECVD, a single 150-nm-thick $\operatorname{SiN}_{x}$ film was grown on a PC substrate at optimized conditions. To compare the WVTR of the passivation layer, 150 -nm-thick $\mathrm{SiN}_{x}$ film was intentionally grown on a PC substrate by ICP-CVD. The WVTR of the single $\mathrm{SiN}_{x}$ film grown by CECVD $\left(0.02-0.06 \mathrm{~g} / \mathrm{m}^{2} /\right.$ day) was found to be much lower than those of the single $\mathrm{SiN}_{x}$ film grown by ICP-CVD $\left(0.1-0.5 \mathrm{~g} / \mathrm{m}^{2} /\right.$ day $)$. The low WVTR of the single $\mathrm{SiN}_{x}$ film grown by CECVD can be attributed to the high density of the $\mathrm{SiN}_{x}$ film caused by the chemical annealing effect of hydrogen atoms in the hydrogen rich ambient. ${ }^{11-14}$

To investigate thermal damage resulting from the tungsten catalyzer with a temperature of $1800{ }^{\circ} \mathrm{C}$ on the electrical and optical properties of the OLEDs, a 150-nm-thick $\mathrm{SiN}_{x}$ passivation layer was deposited over the $\mathrm{Al}$ cathode layer of a test sample. Figure 3(a) shows $J$ - $V$ - $L$ characteristics of the OLED passivated with a $\operatorname{SiN}_{x}$ layer prepared by CECVD and the reference OLED (nonpassivated sample). The $J-V-L$ curve of the OLED passivated with a $\mathrm{SiN}_{x}$ film shows an identical turn on voltage and forward bias behavior to that of the reference OLED. These $J-V-L$ characteristics of OLEDs passivated with a $\mathrm{SiN}_{x}$ film indicate that the electrical and optical characteristics of the OLED are not critically affected by exposure to the high temperature tungsten catalyzer during the $\mathrm{SiN}_{x}$ deposition. In addition, the normalized spectra of the OLEDs passivated with a $\mathrm{SiN}_{x}$ film and reference sample in the inset of Fig. 3(a) indicate that the electron-hole recombination region is still confined to the $\operatorname{Ir}(\text { ppy })_{3}$ doped region after deposition of the $\mathrm{SiN}_{x}$ film by CECVD due to the absence of any thermal damage. Figure 3 (b) shows a comparison of the luminance and power efficiency of the OLED passivated with $\mathrm{SiN}_{x}$ and the reference OLED. As expected from $J-V-L$ results for the OLED, the identical luminance and power efficiency of the OLED passivated with the $\operatorname{SiN}_{x}$ layer to the reference OLED also indicate the potential of CECVD as a thermal damage-free passivation technique. ${ }^{13,14}$ 

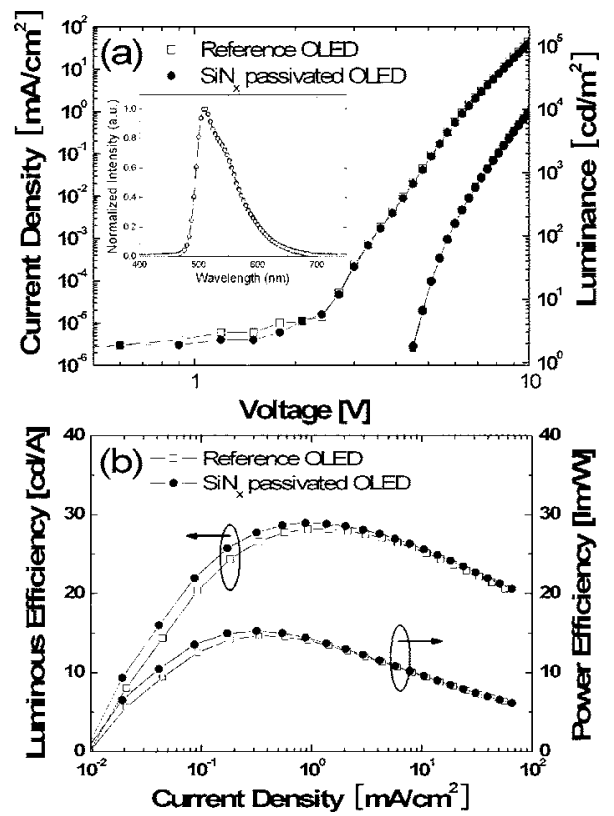

FIG. 3. (a) $J-V-L$ characteristics and (b) efficiency of OLED passivated with 150-nm-thick $\mathrm{SiN}_{x}$ film and nonpassivated OLED (reference sample). The inset is a comparison of the normalized spectra of the OLEDs passivated with a $\mathrm{SiN}_{x}$ film and reference sample.

Figure 4 shows the lifetime and operating voltage for an OLED passivated with 70- and 150-nm-thick $\mathrm{SiN}_{x}$ films and a reference OLED. The lifetime of the reference sample with an initial luminance of $500 \mathrm{~cd} / \mathrm{m}^{2}$ was approximately $53.5 \mathrm{~h}$. The abrupt decrease in the luminance of the reference OLED is related to severe degradation of organic layers by direct intrusion of moisture and oxygen through the cathode layer. ${ }^{2,3}$ However, the lifetime of the OLEDs passivated with 70- or 150 -nm-thick $\mathrm{SiN}_{x}$ film is longer than that of the reference OLED. The lifetime $(\sim 120 \mathrm{~h})$ of OLED passivated with 150 -nm-thick $\operatorname{SiN}_{x}$ is longer than that $(\sim 90 \mathrm{~h})$ of the OLED passivated with 70 -nm-thick $\mathrm{SiN}_{x}$ film, due to longer pathways for the intrusion of moisture and oxygen gas. All OLEDs passivated with $\mathrm{SiN}_{x}$ film above $150 \mathrm{~nm}$ thickness show similar lifetimes because the barrier performance of inorganic film becomes defects limited above certain critical thickness $(100-200 \mathrm{~nm}) .^{6}$ Therefore, the degradation of OLED passivated with $\mathrm{SiN}_{x}$ film can be attributed to the existence of nanodefects $(<1 \mathrm{~nm})$ and the amorphous lattice $(<0.3 \mathrm{~nm})$. Although we cannot find micro- or macrodefects, such as pinhole or cracks at the surface of $\mathrm{SiN}_{x}$ films, nanodefect or amorphous lattice in the $\mathrm{SiN}_{x}$ film can give pathways for the intrusion of moisture and oxygen gas. ${ }^{15}$ In addition, the operating voltage of both reference and $\mathrm{SiN}_{x}$ passivated OLEDs is gradually increased with an increase in operating time due to the intrusion of moisture and oxygen gas into the interface between organic and cathode layers. However, it is noteworthy that the lifetime of the OLED could be prolonged by using a densely grown single $\mathrm{SiN}_{x}$ film unlike other reported thin-film passivation procedures, which use a thick or multilayer film. ${ }^{5,9}$ The prolonged lifetime of the OLED verifies that the $\mathrm{SiN}_{x}$ film grown by CECVD was a very good passivation layer, although a direct comparison of the lifetime with previously reported OLEDs with different types of thin-film passivation layers is difficult because the lifetime of an OLED is significantly dependent on the type of organic materials, structure of the OLED, and

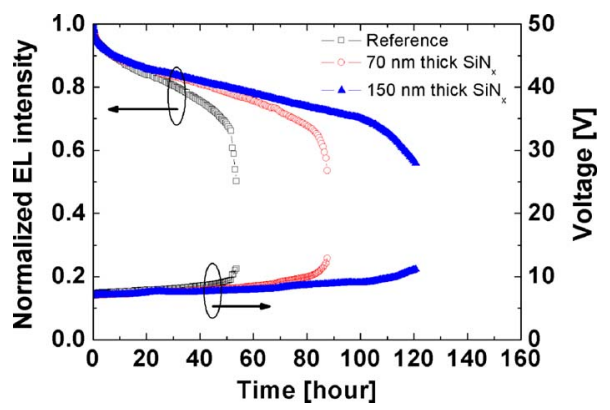

FIG. 4. (Color online) Typical curves for normalized luminance and operating voltage vs operating time of OLEDs passivated with 70- and 150-nm-thick $\mathrm{SiN}_{x}$ films and a reference OLED.

the quality of the OLEDs. Therefore, the identical $J-V-L$ data and prolonged lifetime of the OLED passivated with $\operatorname{SiN}_{x}$ film grown by CECVD suggest that the CECVD technique is a promising plasma-free passivation technique that can be used in place of conventional PECVD and ICP-CVD.

In summary, the passivation properties of $\mathrm{SiN}_{x}$ films grown by CECVD for use in OLEDs were investigated. Using a CECVD equipped with a tungsten catalyzer and BEC system, a high-quality $\operatorname{SiN}_{x}$ film was deposited on PC substrates and a test cell at a substrate temperature of $50{ }^{\circ} \mathrm{C}$. Even at a low substrate temperature, the 150 -nm-thick $\mathrm{SiN}_{x}$ film grown on OLEDs showed superior barrier and optical properties. Due to local substrate heating by hydrogen recombination during $\mathrm{SiN}_{x}$ film deposition, a high-density $\mathrm{SiN}_{x}$ film was produced without any additional substrate heating. These findings indicate that CECVD is a promising plasma-free and low temperature thin-film passivation technique for OLEDs and flexible OLEDs.

This work was supported by Korea Research Foundation Grant funded by Korea Government (MOEHRD: Basic Research Promotion Fund) (KRF-2006-331-D00243) and Ministry of Commerce, Industry and Energy. Experiments at PLS were supported in part by MOST and POSTECH.

${ }^{1}$ C. W. Tang and S. A. VanSlyk, Appl. Phys. Lett. 51, 913 (1987).

${ }^{2}$ P. E. Burrows, V. Bulovic, S. R. Forrest, L. S. Sapochak, D. M. McCarty, and M. E. Thompson, Appl. Phys. Lett. 65, 2922 (1994).

${ }^{3}$ H. Aziz, Z. Popovic, S. Xie, A.-M. Hor, N.-X. Hu, C. Tripp, and G. Xu, Appl. Phys. Lett. 72, 756 (1998).

${ }^{4}$ W. Huang, X. Wang, M. Sheng, L. Xu, F. Stubhan, L. Luo, T. Feng, X. Wang, F. Zhang, and S. Zou, Mater. Sci. Eng., B B98, 248 (2003).

${ }^{5}$ A. B. Chwang, M. A. Rothman, S. Y. Mao, R. H. Hewitt, X. Chu, L. Moro, T. Trajewski, and N. Rutherford, Appl. Phys. Lett. 83, 413 (2003).

${ }^{6}$ M. Schaepkens, T. W. Kim, A. G. Erlat, M. Yan, K. W. Flanagan, C. M. Heller, and P. A. McConnelee, J. Vac. Sci. Technol. A 22, 1716 (2004).

${ }^{7}$ H. Kubota, S. Miyaguchi, S. Ishizuka, T. Wakimoto, Y. Fukuda, T. Watanabe, H. Ochi, T. Sakamoto, T. Miyake, M. Tsuchida, I. Ohshita, and T. Tohma, J. Lumin. 87, 56 (2000).

${ }^{8}$ S. J. Yun, Y.-W. Ko, and J. W. Lim, Appl. Phys. Lett. 85, 4896 (2004).

${ }^{9}$ M. S. Weaver, L. A. Michalski, K. Rajan, M. A. Rothman, J. A. Silvernail, P. E. Burrows, G. L. Graff, M. E. Gross, P. M. Martin, M. Hall, E. Mast, C. Bonham, W. Bennett, and M. Zumhoff, Appl. Phys. Lett. 81, 2929 (2002).

${ }^{10}$ H.-K. Kim, D.-G. Kim, K.-S. Lee, M.-S. Huh, S. H. Jeong, and K. I. Kim, Appl. Phys. Lett. 86, 183503 (2005).

${ }^{11}$ H. Matsumura, Jpn. J. Appl. Phys., Part 1 37, 3175 (1998).

${ }^{12}$ A. Matsuda, Thin Solid Films 337, 1 (1999).

${ }^{13}$ T. Minamikawa, A. Heya, T. Niki, M. Takano, Y. Yonezawa, S. Muroi, S. Minami, A. Masuda, H. Umemoto, and H. Matsumura, Thin Solid Films 501, 154 (2006).

${ }^{14}$ H.-K. Kim, Nucl. Instrum. Methods Phys. Res. B 253, 255 (2006).

${ }^{15}$ A. P. Roberts, B. M. Henry, A. P. Sutton, C. R. M. Grovenor, G. A. D. Briggs, T. Miyamoto, M. Kano, Y. Tsukahara, and M. Yanaka, J. Membr. Sci. 208, 75 (2002). 